\title{
Status and Challenges of the Compressed Baryonic Matter (CBM) Experiment at FAIR
}

\section{Hans Rudolf Schmidt ${ }^{*}$ for the CBM Collaboration}

University of Tuebingen

Auf der Morgenstelle14, Tuebingen, Germany

E-mail: Hans-Rudolf.Schmidt@Uni-Tuebingen.de

The CBM experiment is one of the four scientific pillars of the Facility for Antiprotons and Ion Research (FAIR) in Darmstadt, Germany. Its discovery potential - complementary to heavy-ion experiments at colliders - is based on high-luminosity ion beams. This enables access to extremely rare probes such as charmed particles, vector mesons or multi-strange hyperons with high statistics. However, 3rd generations readout systems and detectors are required to handle the large interaction rates (up to $10 \mathrm{MHz}$ for $\mathrm{Au}+\mathrm{Au}$ ) with sufficient precision and bandwidth. In this contribution we will outline the unique CBM physics program and its relation to other, existing (RHIC-BES, SPS-NA61) or future (NICA-MPD) heavy-ion experiments in a similar energy range (2-35 AGeV). We will further elaborate on the demands and challenges, which are imposed by the high collision rates and event multiplicities on the different CBM detectors systems.

International Winter Meeting on Nuclear Physics

21-25 January 2013

Bormio, Italy

\footnotetext{
${ }^{*}$ Speaker
} 


\section{Introduction}

The observation of a mass $(1.97 \pm 0.04) M_{\odot}$ neutron star [1] has spurred interest into models of nuclear matter capable to stabilize nuclear matter at high densities [2,3,4], i.e., to prevent the collapse of heavy neutron stars into black holes. The relevant degrees of freedom in nuclear matter at high densities are nucleons and hyperons; as well as quarks in the case of the onset of a deconfinement transition. However, experimental information on the properties of hypernuclear or quark matter at low temperature and high baryon density is sparse and theories have to rely on model assumptions [4].

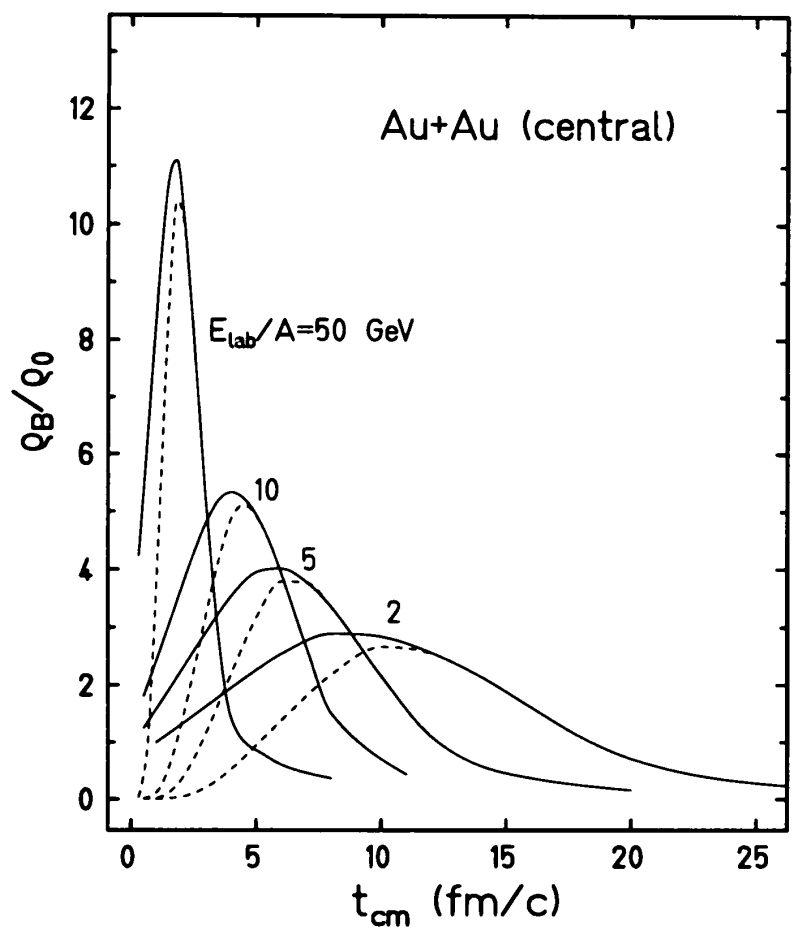

Figure 1: Time evolution of the central baryon density in head-on $\mathrm{Au}+\mathrm{Au}$ collisions for beam energies $2 \leq E_{L a b} \leq 50 \mathrm{AGeV}$. The solid lines correspond to all baryons while the dashed lines are for the participants only [5].

While pretty much understood around saturation density $\rho_{0}$ and somewhat above $[2,6,7,8,9]$, the extrapolation to higher densities $\left(>3 \rho_{0}\right)$ is ambiguous [6,7] and experimental verification is necessary. The future experiments at FAIR (Darmstadt) or at NICA (Dubna) will employ high intensity heavy-ion beams in the range $2-35 \mathrm{AGeV}\left(\sqrt{s_{N N}}=2.7-8.3 \mathrm{GeV}\right)$ and $\sqrt{s_{N N}}=4-$ $11 \mathrm{GeV}$ ), respectively. These experiments will allow to investigate rather exotic matter at baryon densities up to $7 \times \rho_{0}$ (cf. Figure 1) with good statistics for the first time. This will enable to explore yet uncharted territory with rare probes, such as charmed particles, di-leptons, double hypernuclei or multi-strange hyperons [8,9]. It should, however, be noted that the link between nuclear experiments and Neutron Star (NS) observations is a highly complex, nontrivial task $[10,11,12,13,14,15,16,17,18,19,20,21,22]$. 


\section{Experiments probing the high net baryon density regime}

Several experiments are exploring hot and dense matter in various regions of the QCD phase diagram: While the ALICE experiment at the CERN-LHC probes matter exclusively at zero net baryon density, i.e., matter as it was existing a few $\mu s$ after the big bang, the RHIC accelerator allows for experiments at beam energies ranging from $\sqrt{s}=200 \mathrm{AGeV}$ down to $7 \mathrm{AGeV}$. Similarly, the beam energy scan program of the SPS-NA61 (Shine) experiment probes the energy range from $\sqrt{s}=17.4 \mathrm{AGeV}$ down to $6.4 \mathrm{AGeV}$. As can been seen from Figure 2 these lower beam energies allow the exploration of the high net baryon densities (evaluated at freezeout). Shown in Figure 2 is the $T-\rho_{B}$ plane, where $\rho_{B}$ is the net baryon density (equivalent to $\mu_{B}$, the baryo-chemical potential). The maximum freeze-out density is reached around $\mu_{B}=400-500 \mathrm{MeV}$, which corresponds to fixed target energies of $30-40 \mathrm{AGeV}$. It is further worth mentioning that this value of $\mu_{B}$ is expected to be beyond a critical value, above which a deconfinement transition should be first order.

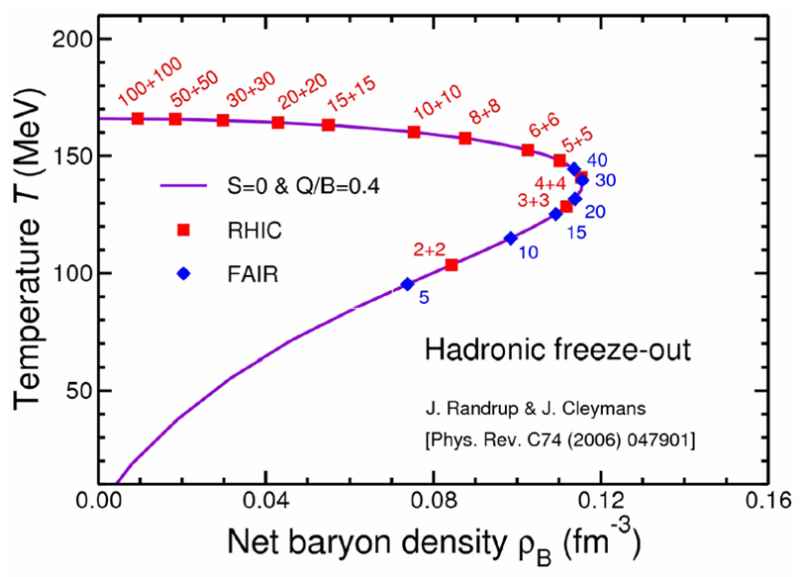

Figure 2: $\quad$ Freeze-out curve from a statistical model analysis in the temperature versus net-baryon density plane, taken from [23]. Red and blue points show the region available by experiments at RHIC and FAIR, respectively, depending on the collision energy.

Both the future experiment MPD@NICA and CBM@FAIR (SIS100/300) will cover an energy range, which will allow exploring the high net baryon density region. However, the beam energy is only one of the crucial parameters for the investigation of dense matter. Of a similar importance is the capability to employ rare probes as messengers of the high density phase. The sensible use of rare probes requires high luminosity beams as well as detectors capable of high rate data taking. Table 1 gives a summary of the beam energy range of the experiments discussed above and their limitations in terms of reaction rates. While collider experiments (RHIC, NICA) are typically luminosity limited - which is particularly true for RHIC at low energies - fixed target experiments can easily increase the reaction rate with target thickness. Their limitations come mainly from the detector capability. In case of the NA61 experiment the TPC is the limiting detector and restricts the data taking to values below $80 \mathrm{~Hz}$. For this reason the future CBM experiment at SIS100/300 is designed as a high rate experiment employing 
state-of-the-art detector technology and - noteworthy - latest computer technology for the data acquisition.

\begin{tabular}{l|c|c|l}
\hline \hline Experiment & $\sqrt{s_{N N}}[\mathrm{GeV}]$ & Reaction Rates[Hz] & Limitation \\
\hline PHENIX, STAR (BNL) & $7-200$ & $1-800$ & luminosity at low $\sqrt{s_{N N}}$ \\
\hline NA61(CERN) & $6.4-17.4$ & 800 & limited by detector (TPC) \\
\hline MPD (NICA) & $4-11$ & 1000 & design lum. $\left(10^{27} \mathrm{~cm}^{-1} \mathrm{~s}^{-1}\right)$ \\
\hline CBM(FAIR) & $2.7-8.3$ & $10^{5}-10^{7}$ & detector technology \\
\hline \hline
\end{tabular}

Table 1: Energy ranges and reaction rates of current (STAR, PHENIX, NA61) and future experiments (MPD, HADES@FAIR, CBM) to explore dense baryonic matter.

\section{Rare Probes}

Rare probes such as low mass di-leptons, charmonium and open charm will allow to characterize the medium at high net baryon densities much more detailed then previously possible. Table 2 shows a compilation of the accessibility of observables for the experiments discussed above. While bulk observables (flow, spectra of hadrons) as well as correlations and fluctuations are accessible to all experiments at the relevant low beam energies $\left(\sqrt{s_{N N}} \sim 8 \mathrm{GeV}\right)$, the above mentioned rare probes can only be measured at CBM@FAIR.

In this paper, however, we will not further focus on leptons and charm. Instead, we will discuss more exotic probes of dense matter: strangeness in the form of multi-strange hyperons, doublestrange hypernuclei and multi-strange di-baryon, the latter also called MEMOs (metastable exotic multi-hypernuclear objects).

\begin{tabular}{l|c|c|c|c|c}
\hline \hline \multirow{2}{*}{ Experiment } & \multicolumn{5}{|c}{ Observables at $\sqrt{S_{N N}}=8 \mathrm{GeV}$} \\
\cline { 2 - 6 } & hadrons & $\begin{array}{l}\text { correlations, } \\
\text { fluctuations }\end{array}$ & di-leptons & $\begin{array}{l}\text { hyperons, double } \\
\text { hypernuclei }\end{array}$ & charm \\
\hline PHENIX, STAR (BNL) & $\sqrt{ }$ & $\sqrt{ }$ & - & - & - \\
\hline NA61(CERN) & $\sqrt{ }$ & $\sqrt{ }$ & - & - & - \\
\hline MPD (NICA) & $\sqrt{ }$ & $\sqrt{ }$ & $(\sqrt{ })$ & $(\sqrt{ })$ & - \\
\hline CBM(FAIR) & $\sqrt{ }$ & $\sqrt{ }$ & $\sqrt{ }$ & $\sqrt{ }$ & $\sqrt{ }$ \\
\hline \hline
\end{tabular}

Table 2: Observables accessible in current and future experiments on dense baryonic matter.

\subsection{Multi-strange Hyperons}

The yields and phase-space distributions of multi-strange hyperons $(\Xi, \Omega)$ are promising tools to study the properties and the degrees of freedom of QCD matter at extreme nuclear densities. In particular, these particles may serve as unambiguous messengers of a high-density phase: for hyperon production binary reactions proceeds like $p p \rightarrow \Xi^{-} K^{+} K^{+} p$ or $p p \rightarrow \Omega^{-} K^{+} K^{+} K^{0} p$ and have accordingly high thresholds of 3.7 and $7.0 \mathrm{GeV}$, respectively. In dense baryonic matter, however, $\Xi^{-}$and $\Omega^{-}$can also be created via strangeness exchange reactions like $\Lambda \Lambda \rightarrow \Xi^{-} p$ and $\Lambda \Xi^{-} \rightarrow \Omega^{-} n$ or $\Lambda K^{-} \rightarrow \Xi^{-} \pi^{0}$, with the $\Lambda$ and the $K^{-}$previously produced in independent 
reactions such as $p p \rightarrow K^{+} \Lambda p$ and $p p \rightarrow K^{+} K^{-} p p$, which require only 1.6.and $2.5 \mathrm{GeV}$, respectively. Alternatively, three-body collisions involving $\Lambda^{\prime}$ s or kaons open new production channels for $\Sigma$ and $\Omega$ with respect to $p p$ reactions. The production of multi-strange hyperons is thus expected to be enhanced at high densities, and their yield to be sensitive to the baryon density reached in the fireball. Moreover, the energy distributions of multi-strange hyperons provide information on the fireball temperature and the radial flow at the time, when they are emitted. Therefore, systematic measurements of $\Xi^{-}$and $\Omega^{-}$production as function of beam energy and size of the colliding nuclei offer the possibility to study the nuclear matter equation of state, or baryon density fluctuations as they are expected to occur when the system undergoes a first-order phase transition. These fluctuations may also indicate the existence and the location of a QCD critical endpoint.

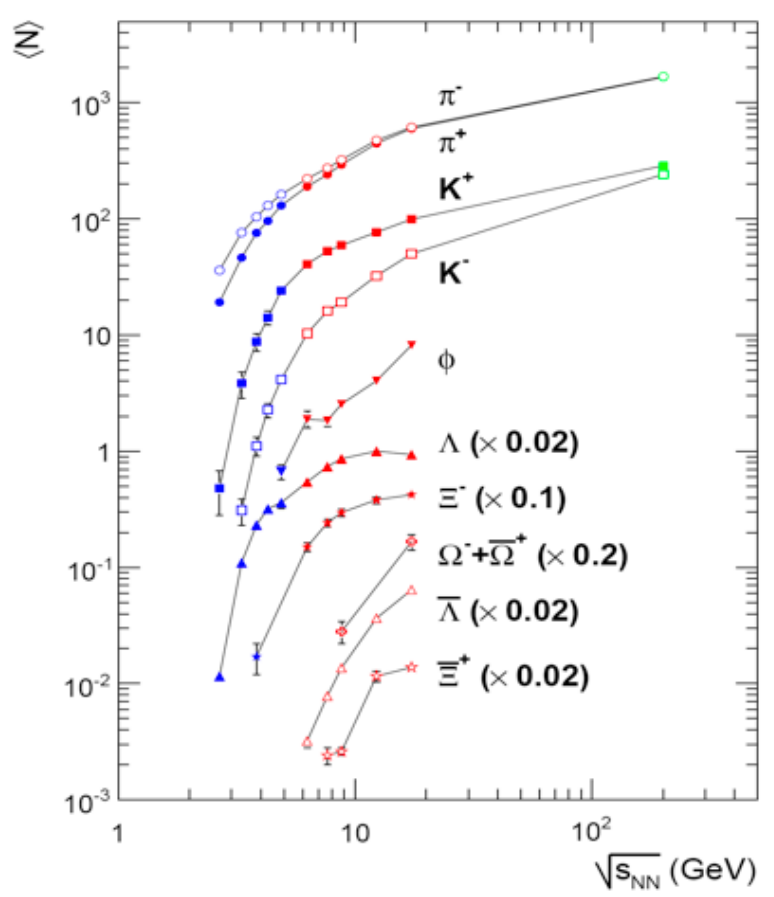

Figure 3: Yield of mesons, hyperons and anti-hyperons as function of collisions energy, measured in central $\mathrm{Au}+\mathrm{Au}$ or $\mathrm{Pb}+\mathrm{Pb}$ collisions (taken from [24])

Existing data on the production of multi-strange hyperons in nuclear collisions at SIS-100 beam energies are scarce as illustrated in Figure 3, where the measured excitation function of strange particles is shown for central collision of heavy nuclei $(\mathrm{Au}+\mathrm{Au}, \mathrm{Pb}+\mathrm{Pb})$ at beam energies above $2 \mathrm{~A} \mathrm{GeV}$ [24]. In particular, no data on production below $40 \mathrm{~A} \mathrm{GeV}\left(\sqrt{S_{N N}}=8.8 \mathrm{AGeV}\right)$ exist, except for $312 \Xi^{-}$measured at $6 \mathrm{AGeV}$ at the AGS [25]. A systematic measurement of multistrange hyperons as diagnostic probes of dense nuclear matter at SIS-100 energies has thus a substantial discovery potential. 


\begin{tabular}{l|c|c|c|c|c}
\hline \hline Energy [AGeV] & $\Sigma^{-}$ & $\Omega^{-}$ & $\bar{\Lambda}$ & $\bar{\Sigma}^{+}$ & $\bar{\Omega}^{+}$ \\
\hline 4.0 & $9.0 \times 10^{6}$ & $1.8 \times 10^{5}$ & $3.6 \times 10^{3}$ & $5.3 \times 10^{3}$ & $9.0 \times 10^{2}$ \\
\hline 6.0 & $2.6 \times 10^{7}$ & $5.0 \times 10^{5}$ & $2.4 \times 10^{5}$ & $1.4 \times 10^{34}$ & $2.8 \times 10^{3}$ \\
\hline 8.0 & $4.0 \times 10^{7}$ & $1.4 \times 10^{6}$ & $3.6 \times 10^{6}$ & $2.0 \times 10^{5}$ & $6.0 \times 10^{4}$ \\
\hline 10.7 & $5.4 \times 10^{8}$ & $2.2 \times 10^{6}$ & $6.8 \times 10^{6}$ & $3.8 \times 10^{5}$ & $1.2 \times 10^{4}$ \\
\hline \hline
\end{tabular}

Table 3: Expected hyperon yields [26] measured per week at a minimum-bias interaction rate of $2 \times 10^{4} s^{-1}$. The minimum bias multiplicity is assumed to be $25 \%$ of the one in central collisions.

A prerequisite for these measurements is sufficient statistics in finite time. Table 3 shows that already at $2 \times 10^{4}$ minimum-bias interactions the accumulated data exceed previous measurements in terms of yield.

\subsection{Search for Double Hypernuclei}

Up to now, only a handful double hypernuclei have been seen, mostly in emulsion experiments. Hypernuclei, i.e. nuclei containing at least one hyperon in addition to nucleons, offer the fascinating perspective to explore the third, strange dimension of the chart of nuclei. Their investigation provides information on the hyperon-nucleon and even on the hyperon-hyperon interactions, which play an important role in neutron star models.

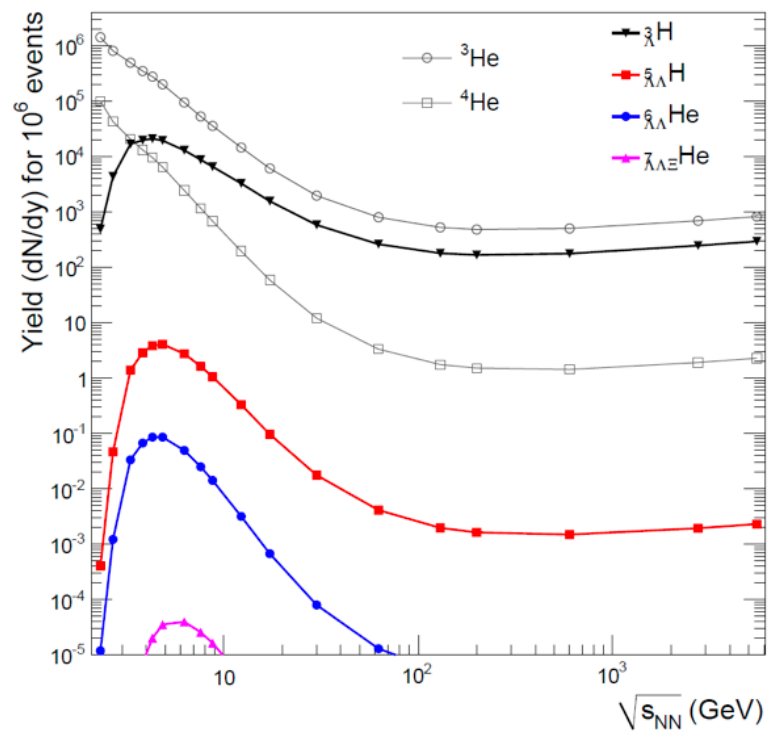

Figure 4: Energy dependence of hypernuclei yields at mid-rapidity for $10^{6}$ central $A u+A u$ events as calculated with the statistical model [27]. The predicted yields of ${ }^{3} \mathrm{He}$ and ${ }^{4} \mathrm{He}$ are included for comparison.

The conventional production mechanism is rather complicated, e.g., via the following chain reaction employing a kaon beam: $K^{-}+N \rightarrow K^{+}+\Xi^{-}, \Xi^{-}+{ }^{12} C \rightarrow{ }_{\Lambda \Lambda}^{6} \mathrm{He}+{ }^{4} \mathrm{He}+t$. Hence the production cross sections are exceedingly small. On the other hand, heavy-ion collisions offer the possibility to produce (double) hypernuclei via coalescence of $\Lambda^{\prime}$ s with nucleons or light fragments in the final state of the reaction. In high-energy nucleus-nucleus collisions, $\Lambda$ 
hyperons are produced abundantly. Their maximum yield is observed at beam energies between 30 and $40 \mathrm{AGeV}$ (about 50 in central $\mathrm{Pb}+\mathrm{Pb}$ collisions). At $10 \mathrm{AGeV}$ still $15 \Lambda^{\prime} \mathrm{s}$ are produced. The coalescence probability of $\Lambda$ with light nuclei is highest at low energies yielding, according to hadron gas model calculations [27], $2 \times 10^{-2}{ }_{\Lambda}^{3} \mathrm{He}, 10^{-6}{ }_{\Lambda \Lambda}^{5} \mathrm{He}$ and $3 \times$ $10^{-8}{ }_{\Lambda \Lambda}^{6} \mathrm{He}$ per central collision at $8-10 \mathrm{AGeV}$. This would give, with the foreseen CBM interactions rates about $120{ }_{\Lambda \Lambda}^{5} \mathrm{He} /$ week and still $3.6{ }_{\Lambda \Lambda}^{6} \mathrm{He} /$ week. For the detection of the double hypernuclei, the decay chain has to be reconstruction in the tracking detectors, e.g., ${ }_{\Lambda}^{5} \mathrm{He} \rightarrow{ }_{\Lambda}^{5} \mathrm{He}+\pi^{-} \rightarrow{ }^{4} \mathrm{He}+\mathrm{p}+\pi^{-}$.

The investigation of double-hypernuclei is also one of the prime goals of the PANDA experiment at FAIR. Even though the focus of PANDA is on the $\gamma$-spectroscopy of double hypernuclei excited states (and not, as for CBM, as a diagnostic tool of high density matter) is intriguing to compare the rates. At the design luminosity $\left(\approx 10^{32} \mathrm{~cm}^{-1} \mathrm{~s}^{-1}\right)$ PANDA expects meaningful spectra after 2 weeks of running in dedicated hypernuclei run [28]. Unfortunately, at SIS100/HESR the luminosity will be about a factor of 10 lower and thus the time to accumulate spectra accordingly longer.

\subsection{Search for Meta-Stable Multi-Strange Objects}

Metastable objects with strangeness, e.g. strangelets and (strange) di-baryons, were proposed long ago as collapsed states of matter, consisting of either baryons or quarks [29,30]. Up to date, none of these objects have been observed. Their existence or absence is an open issue in highenergy physics (cf. [31]).

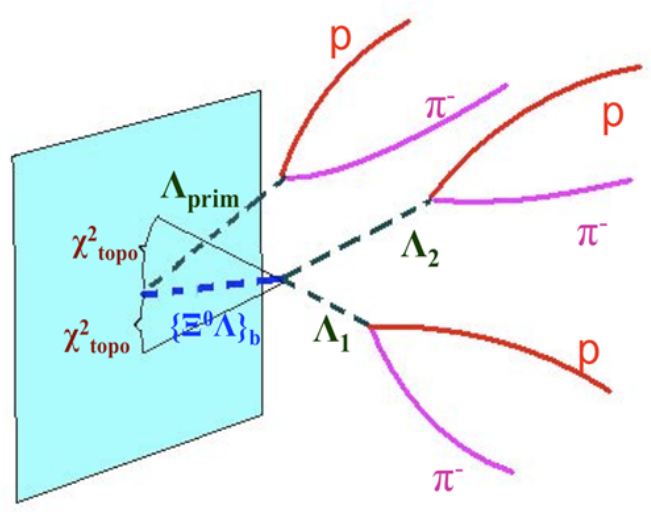

Figure 5: Sketch of the decay topology of a strange di- baryon.

High-energy nuclear collisions, with kaons and $\Lambda^{\prime} s$ being abundantly produced in a single event, could provide a tool to create such composites objects with multiple units of strangeness. For SIS-100 energies, however, no predictions for the multiplicities of bound multi-strange objects are available. At higher energies, calculations with a hybrid (microscopic transport + hydrodynamics) model were performed [32], predicting a multiplicity of about $10^{-2}$ for the $\left(\Xi^{0} \Lambda\right)_{b}$ in central collisions at $30 \mathrm{~A} \mathrm{GeV}$. Owing to the finite lifetime of such an object its decay, e.g., $\left(\Xi^{0} \Lambda\right)_{b} \rightarrow \Lambda \Lambda$, should be detectable via displaced vertices ( $c \tau \approx 1-5 \mathrm{~cm}$ ) [33] as 
shown in Figure 5. Indeed, simulations show that $\left(\Xi^{0} \Lambda\right)_{b}{ }^{\prime} s$ can be reconstructed with a signalto-background ratio above 200 for $10^{12}$ central events [34,35].

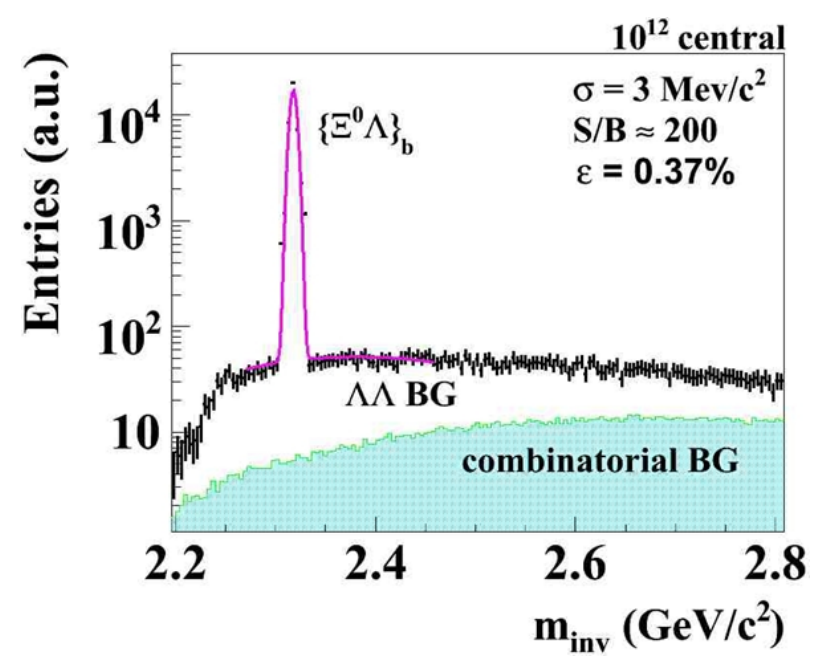

Figure 6: Reconstructed invariant-mass distribution of $\Lambda \Lambda$ candidates

Most of the strange di-baryon searches were done via the $\left(K^{+}, K^{-}\right)$reaction (cf. [36], and Refs. therein), which is not directly comparable with the production of di-baryons in heavy-ion collisions. The nearest equivalent to heavy-ion reactions is a result reported by the KTeV collaboration [37] at Fermilab, were lightly bound $H^{0}$-dibaryons produced in $p N$-collisions were searched for. However, the acceptance of the experimental apparatus was optimal for a decay length $c \tau=160 \mathrm{~cm}$, and thus the reported limit is not applicable for the search range of $c \tau \approx 1-5 \mathrm{~cm}$ accessible in the CBM experiment.

\section{Experimental Setup and Status of Detector Development}

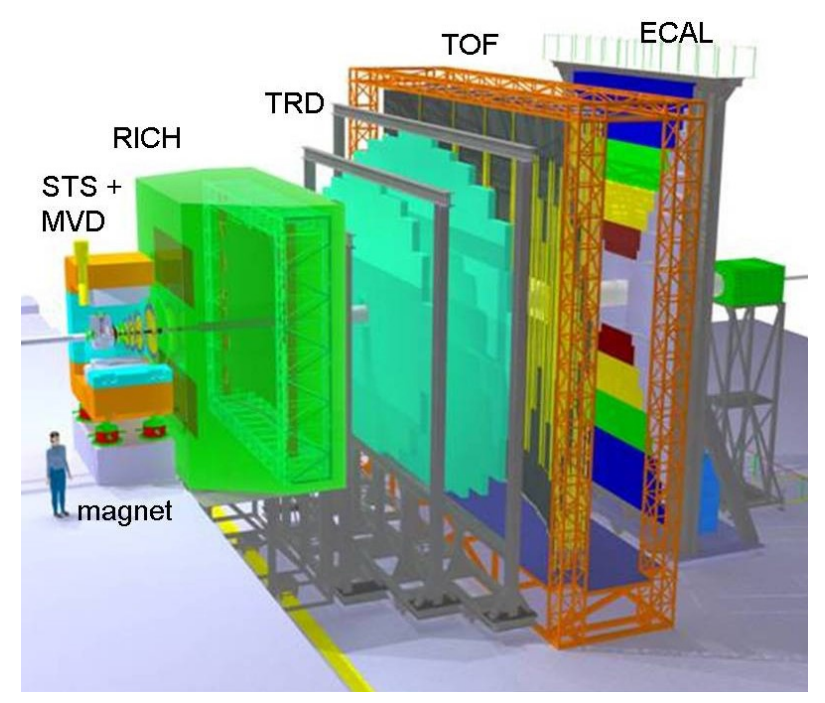

Figure 7: Layout of the di-electron setup of the CBM experiment. The RICH detector is replaced by muon chambers for $J / \Psi-$ detection. 
The measurement of bulk hadrons, and in particular multi-strange hyperons, hypernuclei, lepton pairs and charmed particles in nuclear collisions at SIS-100 energies requires a largeacceptance, high-rate capable detector system. CBM is the first experiment in heavy-physics employing luminosities, which lead to interactions rates comparable to high-energy experiments at the CERN-LHC, and are far beyond present-day heavy-ion experiment capabilities. The prerequisites to perform a high-rate experiment in heavy-ion physics are:

- fast, radiation-hard and rate-capable detectors

- fast and radiation-hard read-out electronics

- high-throughput data acquisition and efficient online data selection

These specifications require new detector technologies as well as innovative front-end readout electronics and online computing. In the following we will give a short overview of the components of the CBM experiment with emphasis on new developments in detector technology.

\section{Silicon Tracking System (STS)}

The main tracking device is an eight-layer silicon detector array located inside a large-aperture super-conducting magnet. Its task is to determine the trajectories of produced particles and to reconstruct hyperons by their decay topology. The R\&D focusses onto the development of radiation hard, low mass, double-sided strip sensors [38].

\section{Micro Vertex Detector (MVD)}

A micro-vertex detector is located directly behind the target aiming for precision measurement of the (displaced) decay vertices of charmed hadrons. The development in CBM concentrates on using Monolythic Active Pixel Sensors (MAPS). The R\&D is on improving the rather modest radiation hardness and readout speed. Significant progress could be made in the recent years [39]. At present, a non-ionizing dose of more then $10^{14} n_{e q} / \mathrm{cm}^{2}$ is within reach, which goes even beyond the CBM requirements. The detectors are typically thinned down to only $50 \mu \mathrm{m}$, resulting (including support, cooling and cables) into a material budget of only a few $0.1 \% X_{0}$, which correspond to the CBM requirements. The achieved readout speed allows rates above $100 \mathrm{kHz}$, which is sufficient for charm reconstruction. The spatial resolution is below $3.5 \mu m(\sigma)$.

\section{RICH and TRD}

These two detectors are designed to achieve a combined $\pi$-misidentification factor of about $10^{4}$. The RICH (Ring Imaging Cerencov Hodoscope) features a $\mathrm{CO}_{2}$ radiator, a segmented mirror and MAPMT-photo detection. A full size prototype has been successfully tested in 2012 at the CERN-PS and achieved a $7 \sigma e^{-} / \pi$ separation at $8 \mathrm{GeV} / c$ [40]. The R\&D for the TRD (Transition Radiation Detector) concentrates on geometrical modifications to increasing the rate capabilities while keeping a good $e / \pi$-discrimination. 


\section{RPC for Time-of-Flight}

The R\&D on RPC's (Resistive Plate Chamber) focusses on technologies to improve the rate capabilities. While multi-gap RPC's, as installed in the ALICE and FOPI experiments, deliver excellent timing resolution, they are inherently slow due to the float glass used as resistive plates. New ceramic RPC's with a resistivity of $10^{9} \Omega \mathrm{cm}$ were investigated with test beams and show a rate capability of $10^{4}-10^{5} \mathrm{~cm}^{-2} \mathrm{~s}^{-1}$ in accordance with the CBM requirements. At the same time a timing resolution of the order of $80-100$ ps is achieved [41].

\section{Muon Chambers}

Iron absorbers instrumented with GEMs (Gas Electron Multiplier) are developed by the CBM collaboration for the muon detection. The expected rates are up to several $\mathrm{MHz} / \mathrm{cm}^{2}$. GEMs are chosen because of their known high rate capability and ageing properties. However, GEMs have up to know not been used in the harsh environment of a hadron absorber. The stability of triple GEM detectors under a high (hadronic) radiation is thus under intense R\&D [42].

\section{Data Acquisition and Online Event Reconstruction}

At the design interaction rate $\left(10^{7}\right.$ events $\left./ s\right)$, the expected raw data flow from the front-end electronics (FEE) is about $1 \mathrm{~TB} / \mathrm{s}$, which has to be reduced to the archival rate of about $1 \mathrm{~GB} / \mathrm{s}$ by online data selection. The high rates and complicated trigger patterns (e.g., open charm decays) forbid the use of conventional, latency-limited triggers. Instead, the FEE will be selftriggered, i.e., autonomously streaming hit information with time stamps through the data acquisition chain to a first-level event selector (FLES), The DAQ speed is increased by several orders of magnitude by the usage of these features [43]. With the current software performance, we estimate the required size of the FLES farm to be of the order of 60000 cores.

\section{Summary and Outlook}

Heavy-ion collisions in the SIS-100/300 energy range are an ideal tool for the production of hadronic matter at neutron star core densities, and hence offer the unique opportunity to investigate fundamental properties of strongly interacting systems and its constituents: the nuclear matter equation of state, exotic new phases such as quarkyonic matter, in-medium modifications of hadrons as a signature for chiral symmetry restoration, hypernuclei and multistrange objects, charm production at threshold beam energies, and charm propagation in nuclear matter.

The foreseen high rate capability of the CBM experiment requires intense R\&D for all detector systems. During the last year significant progress has been made resulting into presently four Technical Design Reports (STS, RICH, PSD ${ }^{2}$ and super-conducting magnet).

\footnotetext{
${ }^{2} \mathrm{PSD}=$ Projectile Spectator Detector
} 


\section{References}

[1] P. B. Demorest, T. Pennucci, S. M. Ransom, M. S. E. Roberts, and J. W. T. Hessels, Nature 467, 1081 (2010).

[2] I. Sagert, L. Tolos, D. Chatterjee, J. Schaffner-Bielich and C. Sturm, Phys. Rev. C 86, 045802 (2012)

[3] M. Orsaria, H. Rodrigues, F. Weber, and G. A. Contrera, Phys. Rev. D 87, 023001 (2013)

[4] G. Colucci and A. Sedrakian, arXiv:1302.6925 (2013)

[5] B. Friman, W. Nörenberg and V. D. Toneev, Eur. Phys. J. A 3 (1998) 165

[6] B. Li, L. Chen, and C. M. Ko, Phys. Rep. 464, 113 (2008).

[7] B.-A. Li and A. W. Steiner, Phys. Lett. B 642, 436 (2006).

[8] B. Frimann et al. (eds.), "The CBM Physics Book", Springer Lecture Notes in Physics 814 (2011)

[9] P. Senger and V. Friese, https://www.gsi.de/documents/DOC-2011-Aug-29.html

[10] G. Q. Li, C.-H. Lee, and G. E. Brown, Phys. Rev. Lett. 79, 5214 (1997).

[11] T. Klahn, D. Blaschke, S. Typel, E. N. E. van Dalen, A. Faessler, C. Fuchs, T. Gaitanos, H. Grigorian, A. Ho, and Kolomeitsev et al., Phys. Rev. C 74, 035802 (2006).

[12] D. H. Youngblood, H. L. Clark, and Y.-W. Lui, Phys.Rev. Lett. 82, 691 (1999).

[13] J.M. Lattimer, M. Prakash, Science 304, 536 (2004)

[14] F. Weber, Pulsars as astrophysical laboratories for nuclear and particle physics (IOP, Bristol, UK, 1999)

[15] D. Blaschke, N.K. Glendenning, A. Sedrakian (eds.), Physics of Neutron Star Interiors, Lecture Notes in Physics, vol. 578 (Springer, Berlin, 2001)

[16] F. Weber, Prog. Nucl. Part. Phys. 54, 193 (2005)

[17] D. Page, S. Reddy, Ann. Rev. Nucl. Part. Sci. 56, 327 (2006)

[18] F. Weber, R. Negreiros, P. Rosenfield, M. Stejner, Prog. Nucl. Part. Phys. 59, 94 (2007)

[19] J.M. Lattimer, M. Prakash, Phys. Rept. 442, 109 (2007)

[20] J. Schaffner-Bielich, PoS CPOD07, 062 (2007)

[21] H. Heiselberg, V. Pandharipande, Ann. Rev. Nucl. Part. Sci. 50, 481 (2000)

[22] J. Piekarewicz, AIP Conf. Proc. 1128, 144 (2009)

[23] J. Randrup and J. Cleymans, Phys. Rev. C 74 (2006) 047901

[24] C. Blume, J. Phys. G 31 (2005) S57

[25] P. Chung et al., Phys. Rev. Lett. 91, 202301 (2003)

[26] A. Andronic, private communication

[27] A. Andronic et al., Phys. Lett. B 697 (2011) 203

[28] Physics Performance Report, arxiv.org/abs/0903.3905v1(2009)

[29] A. R. Bodmer, Phys. Rev. D 4 (1971) 1601

[30] R. L. Jaffe, Phys. Rev. Lett. 38 (1977) 195

[31] H. Clement, Prog. Part. Nucl. Phys. 67, 486 (2012)

[32] H. Stöcker et al., Nucl. Phys. A 827 (2009) 624c

[33] J. Schaffner-Bielich, R. Mattiello and H. Sorge, Phys. Rev. Lett. 84 (2000) 4305

[34] I.Vassiliev, private communication

[35] I. Vassiliev, I. Kisel, and D. Malakhov, CBM Progress Report 2009

[36] R.W. Stotzer et al, Phys. Rev. Lett. 79, 3646 (1997)

[37] A.Alavi-Harati et al., Phys. Rev. Lett. 84, 2593 (2000)

[38] J. Heuser et al., CBM Progress Report 2011

[39] D. Doering, M. Deveaux, M. Domachowski, I. Fröhlich, M. Koziel, C. Müntz, P. Scharrer, and J. Stroth, CBM Progress Report 2011

[40] J. Kopfer et al., CBM Progress Report 2011

[41] B. Kämpfer et al., CBM Progress Report 2011

[42] S. Biswas et al., CBM Progress Report 2011

[43] J. de Cuveland, D. Hutter, and V. Lindenstruth, CBM Progress Report 2011 